\title{
What Observation of Motor Skills Does and Does Not Teach Us
}

\author{
Nicola J Hodges ${ }^{*} \quad$ Nicole T Ong ${ }^{*} \quad$ Beverley C Larssen* Shannon B. Lim* \\ (*)School of Kinesiology, University of British Columbia, Canada \\ E-mail: nicola.hodges@ubc.ca,nicole.ong@alumni.ubc.ca,blarssen@interchange.ubc.c, \\ shannonblim@gmail.com
}

\begin{abstract}
We review data from 4 experiments where we have been studying what is learnt through observation. In these experiments people learnt to reach in a distorted visual-motor, virtual environment. In all experiments observers successfully adapted to new visual-motor environments just by watching. Importantly, however, they adapted differently to actors. At no time did nä̈ve observers show after-effects when returned to a known normal environment. However, if observers had previously practiced in this environment, after-effects were subsequently seen following an observation phase. Further, again different to actors, they showed good retention and lack of interference when performing in two opposing environments. We argue that observation does not result in the updating of an internal (motor) model, that it is primarily strategically mediated and that only after physical experience in the environment can 'motor-simulation' through observation take place.
\end{abstract}

\section{Introduction}

Learning through watching has long been considered a useful practice method and is probably one of the most favoured and widely used techniques in the teaching of motor skills [1]. Much of learning, via this observation method, is expected to occur via explicit, strategic means [2]. Physical practice is believed necessary to translate the acquired information into a motor code (late mediation hypothesis [3]).

However, there have been claims that observational learning engages motor-related (implicit) processes during the observation stage and that this activation of the motor system during observation encourages learning in a similar manner to physical practice $[4,5]$. Mechanistically, neurophysiological research showing shared activations in areas of the brain involved in both action execution and action observation [6] have provided the impetus for these claims.
In one key study that relates to our experiments [4], researchers showed the effectiveness of observational practice for learning a dynamic (robot-administered) adaptation task. Observers watched an actor adapt to a novel force-field environment during reaching actions. Following observation, positive/direct learning effects were seen when the observers performed in this novel environment. Although the authors presented evidence that this adaptation was mediated by implicit/motorrelated processes, they did not test for after-effects after observation in a normal environment. In the adaptation learning literature, this is a key effect necessary to show that there has been a change in the motor control processes (i.e., internal modeling) that are somewhat impermeable to conscious/explicit control. This would provide strong evidence that observation and physical practice both engage motor processes and that learning proceeds by similar mechanisms in both.

Therefore, in subsequent experiments we used a visuomotor, virtual environment, where participants aimed to targets with trajectory feedback that was rotated by $30 \mathrm{deg}$ in a clockwise (CW) or counterclockwise (CCW) direction. In addition to testing for after-effects following observation (Exp1) and observation + imagery $(\operatorname{Exp} 2)$, we tested whether there would be advantages associated with observational practice over physical practice. In previous research, actors showed significant (retroactive) interference in retaining a model of performance in one environment (e.g., $\mathrm{CW}$ ) following practice of a second rotation (e.g., CCW, [7]). Arguably, these effects were related to difficulties in holding multiple internal models of the environment. If observation does not promote the updating of an internal model (i.e., motor-based simulation), then we should not see this interference in observers (Exp 3$)$. In a final experiment we tested for after-effects in observers who had previously received physical practice but after-effects had been washed-out before a subsequent observation phase. If action simulation is only possible once the action is part of the person's

This is an Open Access article distributed under the terms of the Creative Commons Attribution-Noncommercial License 3.0, which permits unrestricted use, distribution, and reproduction in any noncommercial medium, provided the original work is properly cited. 
Table 1: Experimental phases and associated observational practice and visuomotor environmental conditions/trials as a function of Experiment and group $(\mathrm{OBS}=$ observer, $\mathrm{ACT}=$ actor, $\mathrm{H}=$ hand vision, $\mathrm{NH}=$ hand vision occluded $(\mathrm{C}) \mathrm{CW}=($ counter $)$ clockwise $)$.

\begin{tabular}{|c|c|c|c|c|c|c|c|}
\hline Group/Phase & $\begin{array}{l}\text { Pretest } \\
(\mathrm{t}=50)\end{array}$ & $\begin{array}{l}\text { Adapt } 1 \\
(\mathrm{t}=150-200)\end{array}$ & $\begin{array}{l}\text { Posttest } 1 \\
\text { Aftereffects } 1 \\
(\mathrm{t}=50)\end{array}$ & $\begin{array}{l}\text { Adapt } 2 \\
(\mathrm{t}=150)\end{array}$ & $\begin{array}{l}\text { Posttest } 2 \\
\text { Aftereffects } 2 \\
(t=50)\end{array}$ & $\begin{array}{l}\text { Posttest } 3 \\
\text { Direct effects } 1 \\
(\mathrm{t}=50)\end{array}$ & $\begin{array}{l}\text { Posttest } 4 \\
\text { Direct effects2 } \\
(\mathrm{t}=50)\end{array}$ \\
\hline \multicolumn{8}{|l|}{$\operatorname{Exp} 1(n=10 / g p)$} \\
\hline OBS & normal & WatchCW & $X$ & $X$ & normal & $\operatorname{actCW}$ & $X$ \\
\hline ACT_H & normal & ActCW & $\mathrm{X}$ & $X$ & normal & $\mathrm{X}$ & $X$ \\
\hline ACT_NH & normal & ActCW & $X$ & $\mathrm{X}$ & normal & $X$ & $X$ \\
\hline \multicolumn{8}{|l|}{$\operatorname{Exp} 2(n=10 / g p)$} \\
\hline OBS+Image & normal & WatchCW & $X$ & $X$ & normal & $\operatorname{actCW}$ & $X$ \\
\hline ACT_H & normal & ActCW & $\mathrm{X}$ & $\mathrm{X}$ & normal & $\mathrm{X}$ & $\mathrm{X}$ \\
\hline \multicolumn{8}{|l|}{$\operatorname{Exp} 3(n=8 / g p)$} \\
\hline OBS & normal & ActCW & $X$ & WatchCCW & $\mathrm{X}$ & ActCW & ActCCW \\
\hline ACT-NH & normal & ActCW & $X$ & ActCCW & $\mathrm{X}$ & ActCW & ActCCW \\
\hline \multicolumn{8}{|l|}{$\operatorname{Exp} 4(n=9)$} \\
\hline Act_OBS & normal & ActCW & $\begin{array}{l}\text { normal } \\
\text { (+ washout) }\end{array}$ & WatchCW & normal & $\mathrm{X}$ & $X$ \\
\hline
\end{tabular}

motor repertoire we would now expect after-effects following observation (Exp 4).

\section{Methods}

\section{1}

\section{Participants and groups}

In all experiments participants were recruited from the University of British Columbia and they were paid $\$ 8 /$ hour for participating. All procedures were conducted according to the ethical guidelines of the University (see Table 1).

\section{2}

\section{Task and apparatus}

Participants were required to guide a cursor to aim successively to 5 targets projected in a virtual environment, whereby the cursor was rotated $30 \mathrm{deg}$ clockwise, CW (and counterclockwise, CCW Exp 3). Aiming accurately to a target required a fast, aiming movement 30 deg $\mathrm{CCW}$ to the target (participants were not told this). The apparatus and stimuli have been reported elsewhere [8-10]. Participants sat in a chair facing a horizontal, semi-silvered mirror, fixed above a Calcomp graphics' tablet that measured 2D displacement. A monitor, $30 \mathrm{~cm}$ above the mirror, reflected an image of the visual stimuli and cursor onto the mirror. The cursor was controlled by a pointing device that was attached to the right index finger. The visual stimuli consisted of a central starting square and 5 radially arrayed targets that were presented $10 \mathrm{~cm}$ from the starting square. Targets were separated by $72^{\circ}$, starting at location $0^{\circ}$ through $288^{\circ}$.

Observers watched a gender-neutral video display of either a paired actor-learning model (Exp $1 \& 3)$ or a trained model (Exp 2 \& 4). Observers were immersed in the environment of the actor, such that they sat in the chair facing the same mirror set-up as actors with the video display reflected off the semi-silvered mirror. In Exp 1 a laptop video display was used.

\subsection{Procedures}

The experiments were generally divided into three phases; pretest, adaptation, posttests (Table 1). At the start of experimentation, all participants were given the opportunity to familiarize to the general task in a normal environment, where the movement of the cursor corresponded directly to the hand movement. After familiarization, participants were pretested in the normal environment without cursor or hand vision.

All groups were instructed before adaptation phases (whether watching or performing) that the forthcoming environment was different from the normal environment and that the response of the cursor to hand movement had changed. During adaptation, participants were either physically exposed to the new 
environment (30 deg CW or CCW, Exp 3) or watched a video of an actor on a laptop (Exp 1) or reflected on to the mirror (Exp2-4). The actor's objective was to direct the cursor fast and accurately to the target.

Actor groups received $\sim 150$ trials of physical practice. For some groups, vision of the hand was also available during these trials (see Table 1). Rests were given after 50 trial blocks. Observers were informed that they would be tested in the same environment as the actor. The observers in Exp 2 were also instructed to imagine that it was their hand they viewed and to try and feel that it was their hand that moved as they watched the video (to encourage 'simulation').

After adaptation, a normal posttest $(\mathrm{t}=50)$ was conducted to test for after-effects. These were conducted without vision of the cursor or hand. Participants were told that the environment was normal (in Exp 4 a normal environment, "washout" period, with real cursor feedback was also given). We then tested for direct evidence of learning/retention of the watched novel environment in posttests (including the CCW environment in Exp 3).

\section{4 \\ Data analysis}

Data collection, filtering and derivation of spatial/kinematic information were identical to procedures reported in Ong and Hodges [8]. Movement direction was measured as the angle from the origin (middle of the start square) to the position of the cursor at peak tangential velocity. The key independent variable, directional error from the intended target, was calculated as the difference between movement direction at peak tangential velocity and target location. A positive value or negative value for error denoted a CW or CCW directional error respectively.

Movement trials that exceeded $300 \mathrm{~ms}$ were excluded (Exp2-4). Mean directional constant error is reported for all analyses. Statistical comparisons involved mixed-factor ANOVAs performed for aiming movements in the normal visuomotor conditions (i.e. pretest, posttest/after-effects) as well as under rotated (adapted) conditions, that is, across early or late practice for the actor (ACT) groups and in posttests/direct-effects for the observer (OBS) groups. Group was the between factor, while Time (pre-post / early adaption-late adaption) were within factors.

\section{Results}

In Figure 1 we have presented results from participants in Exp. 1, 2 and 4 pertaining to pre-test errors and the presence/absence of after-effects (all tested in normal environments). On the far right of the figure we have also shown errors corresponding to direct (learning) effects when the observers and actors (first block of acquisition) were first tested in the perturbed environment. Although not shown, all the actors improved significantly during physical practice $(p s<.01)$. Significant interaction effects in Exp $1 \& 2$ $(p s<.01)$ were a result of an increase in error for actors from pre to post (after-effects test), but not for observers $[8,10]$. Only when practice experience preceded the observation phase (Exp 4) were small after-effects seen (pre vs. post, $p<.05$, Obs4, Fig 1).

Observation aided adaptation, as evidenced by reduced errors for observers when first exposed to the adapted environment, compared to actors $(p s<.05)$.

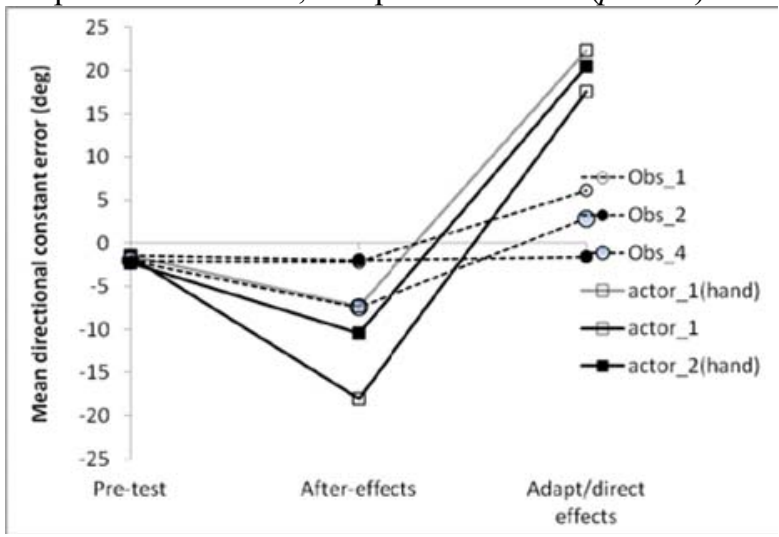

Figure 1: Mean directional constant error (deg) as a function of test. Pre-test and after-effects were informed normal-aiming conditions. Adapt/direct effects shows first exposure to the $30 \mathrm{deg} \mathrm{CW}$ environment. Data are presented from 3 observer groups (circles) across 3 experiments (Obs 1,2 \& 4) and from 3 actor groups (squares), both with vision of their hand and without, in Exp. 1 (actor1 (hand), actor1) and 2 (actor2 (hand)). Even though observers showed benefits from observing when first tested in the new environment, evidence of after-effects were only shown if previous experience was provided.

Data for Observers and Actors from Exp. 3 [9] are shown in Fig 2. Observation did not cause interference when observers were retested in the $\mathrm{CW}$ environment, opposite to actors $(p<.01)$. However, observers learnt from watching, showing less error than actors in $\mathrm{CCW}$ environment $(p<.05)$. This was further confirmed by no $\mathrm{CCW}$ practice control group comparisons (not shown).

\section{Discussion}

In four experiments we have shown that observation encourages a different type of learning than physical practice. Although it can lead to similar effects (aiding 
movements in a novel environment), unless the person can already perform in that new environment (Exp 4), observational practice appears driven by a different, non-motorically-driven, process than physical practice.

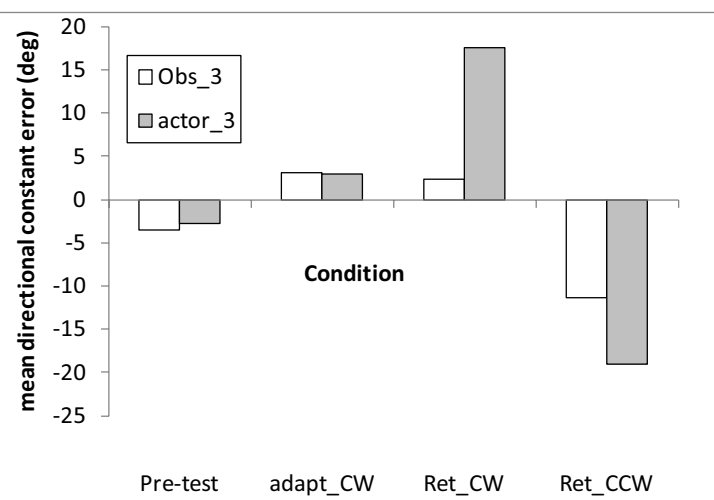

Figure 2: Mean directional constant error (deg) as a function of condition in Exp3 [9]. Errors were low in the pre-test (normal) and in the last 2 blocks of adaptation to the $\mathrm{CW}$ rotation (adapt_CW). Following either observation or physical practice of the CCW rotation (not shown), only the actors showed high errors when re-tested under CW conditions (Ret_CW), even though the observers had learnt the observed rotation (Ret_CCW).

Although there may be disadvantages associated with observational practice in comparison to acting, in these experiments we have also shown advantages associated with this qualitatively different type of practice. Not only are unintentional, negative aftereffects not seen for observers, despite significant direct improvements in the adapted environment (Exp 1 \& 2), it is possible for observers to simultaneously hold models of two opposing environments in memory, with little interference between them (Exp 3). This is in contrast to significant interference for physical practice groups. These data suggest that observational learning allows for independent storage of different models of the environment, perhaps because it does not lead to an updating of an internal model or it allows for multiple, yet distinct (perhaps externally-referenced) models.

We have evidence that observers acquire more explicit/strategic knowledge as a result of watching rather than doing $[8,10]$, but this knowledge, in and of itself is not enough to prevent after-effects. Although our observers showed significantly more evidence of the direction and size of the rotation, this knowledge did not differ from actor groups who learnt with vision of their hand and whom still showed after-effects [8].

We conclude that observation does not automatically involve activation of motor-related systems, unless the motor-skills are an already existing part of the observer's motor repertoire (Exp 4; [11]). We show evidence that for learning, observational practice involves different learning routes or networks to those involved in physical practice, not involving the updating of an internal model of the visual-motor environment (that is the relations between motor commands and sensory inputs). What is more, we show that this observational practice methods can actually be more beneficial for learning than physical practice, when multiple 'skills' are required.

\section{References}

[1] D. Maslovat, S.J. Hayes, R. Horn, and N.J. Hodges. Motor learning through observation (pp 315 -340). In D. Elliott \& M.A. Khan (Eds). Vision \& Goal-Directed Movement. Champaign, Il: Human Kinetics, 2010.

[2] N.J. Hodges, and I.M. Franks. Instructions, demonstrations and the learning process: creating and constraining movement options (pp 145 - 174). In A.M. Williams \& N.J. Hodges (Eds). Skill Acquisition in Sport. London, UK: Routledge, 2004.

[3] S. Vogt, \& R. Thomaschke. From visuo-motor interactions to imitation learning: Behavioural \& brain imaging studies. Jn of Sports Sciences, 25, 497-517, 2007.

[4] A. Mattar, \& P. Gribble. Motor learning by observing. Neuron, 46, 153-160, 2005.

[5] C. Heyes, \& C. Foster. Motor learning by observation: Evidence from a serial reaction time task. Q Jn of Experimental Psychology, 55, 593-607, 2002.

[6] V. Gallese, L. Fadiga, L. Fogassi, \& G. Rizzolatti. Action recognition in the premotor cortex. Brain, 119, 593-609, 1996.

[7] J. Krakauer, M. Ghilardi, \& C. Ghez. Independent learning of internal models for kinematic \& dynamic control of reaching. Nat Neurosci, 2, 1026-1031, 1999. [8] N. Ong \& N.J. Hodges. Absence of after-effects for observers after watching a visuomotor adaptation. Experimental Brain Research, 205, 325-334, 2010.

[9] B. Larssen, N. Ong, \& N.J. Hodges. Watch and learn: Seeing is better than doing when acquiring consecutive motor tasks (in review).

[10] N. Ong, B. Larssen \& N.J. Hodges. Physical practice is needed to promote implicit learning processes and after-effects following observation and imagery (in review).

[11] B. Calvo-Merino, J. Grèzes, D. Glaser, R. Passingham, \& P. Haggard. Seeing or doing? Influence of visual and motor familiarity in action observation. Current Biology, 16, 1905-1910, 2006.

This research is supported by NSERC (Natural Sciences \& Engineering Research Council of Canada) 